Pacific Journal of Mathematics

SHARPNESS IN YOUNG'S INEQUALITY FOR CONVOLUTION 


\title{
SHARPNESS IN YOUNG'S INEQUALITY FOR CONVOLUTION
}

\author{
JOHN J. F. FOURNIER
}

Let $p$ and $q$ be indices in the open interval $(1, \infty)$ such that $p q<p+q$; let $r=p q /(p+q-p q)$. It is shown here that there is a constant $C_{p, q}<1$ such that, if $G$ is a locally compact, unimodular group with no compact open subgroups, and if $g$ and $f$ are functions in $L^{p}(G)$ and $L^{q}(G)$ respectively, then

$$
\|g * f\|_{r} \leqq C_{p, q}\|g\|_{p}\|f\|_{q} ;
$$

here $g * f$ denotes the convolution of $g$ and $f$. Thus, in this case, Young's inequality for convolution is not sharp; this result is used to prove a similar statement about sharpness in Kunze's extension of the Hausdorff-Young inequality. The best constants in these inequalities are known in many special cases; the methods used here do not yield good estimates for these constants, but they do lead to the first proof of nonsharpness for general unimodular groups without compact open subgroups.

1. Introduction. Throughout this paper, we assume that $G$ is a locally compact, unimodular group. We call a pair of indices $(p, q)$ internal if

$$
p>1, q>1, \quad \text { and } \quad \frac{1}{p}+\frac{1}{q}>1 .
$$

Given an internal pair of indices $(p, q)$, we denote by $r$ the index such that

$$
\frac{1}{r}=\frac{1}{p}+\frac{1}{q}-1 .
$$

It is known [17, pp. 54-55; 8, Theorem 20.18] that if $g \in L^{p}(G)$ and $f \in L^{q}(G)$, then the convolution $g * f$ is finite almost everywhere, and measurable; moreover,

$$
\|g * f\|_{r} \leqq\|g\|_{p}\|f\|_{q}
$$

This inequality was first proved, for the circle group, by W. H. Young [18]. Given a group $G$, and an internal pair of indices $(p, q)$, we let

$$
c_{p, q}(G)=\sup \left\{\|g * f\|_{r}: g \in L^{p}(G), f \in L^{q}(G),\|g\|_{p} \leqq 1,\|f\|_{q} \leqq 1\right\} .
$$

Inequality (1) states that $c_{p, q}(G) \leqq 1$. In Theorem 1 of $\S 2$ of this 
paper, we show that, for each internal pair of indices $(p, q)$, there exists a constant $C_{p, q}<1$ such that, if $G$ has no compact open subgroups, then $c_{p, q}(G) \leqq C_{p, q}$.

We call a pair of measurable functions $(g, f)$ maximal if there is an internal pair of indices $(p, q)$ such that $0<\|g\|_{p}\|f\|_{q}<\infty$, and equality holds in inequality (1). A function $\phi$ on $G$ is called a $s u b$ character if there exists a compact open subgroup $H$ of $G$, and a continuous homomorphism $\chi$ of $H$ into the circle group, such that $\phi$ vanishes off $H$, and $\phi$ coincides with $\chi$ on $H$. In Theorem 3 of $\S 3$, we show that a pair of functions $(g, f)$ is maximal if and only if there is a subcharacter $\phi$ such that $g$ and $f$ coincide, almost everywhere, with multiples, by nonzero constants, of left and right translates of $\phi$ respectively.

To discuss R. Kunze's extension of the Hausdorff-Young theorem, we must recall a few facts from the theory of noncommutative integration; this theory is developed in [15], [16], [9], and [10]. Let $f \in L^{p}(G)$ for some $p$, and let $L_{f}$ be the, possibly unbounded, operator on $L^{2}(G)$ given by convolution on the left with $f$; that is, $L_{f}: h \mapsto f * h$. If $L_{f}$ is a bounded operator on $L^{2}(G)$, let $\left\|L_{f}\right\|_{\infty}$ be the norm of this operator; otherwise, let $\left\|L_{f}\right\|_{\infty}=\infty$. If $L_{f}$ is a projection, define $m\left(L_{f}\right)$ to be the extended real number $\|f\|_{2}^{2}$; this functional $m$ can then be extended [15] to a canonical gage on an appropriate set of positive operators so that

$$
m\left(\left|L_{f}\right|^{2}\right)=\|f\|_{2}^{2},
$$

for all $f$ in $L^{2}(G)$. Finally, for each index $q$ with $1 \leqq q<\infty$, let $\left\|L_{f}\right\|_{q}=m\left(\left|L_{f}\right|^{q}\right)^{1 / q}$. It follows from these definition that

$$
\left\|L_{f}\right\|_{\infty} \leqq\|f\|_{1}, \quad \text { and } \quad\left\|L_{f}\right\|_{2}=\|f\|_{2},
$$

for all $f$. Now Kunze showed that, if $1 \leqq p \leqq 2$, then

$$
\left\|L_{f}\right\|_{p^{\prime}} \leqq\|f\|_{p} \text {. }
$$

Here, as in the rest of this paper, $p^{\prime}$ denotes the index conjugate to $p$; that is, $1 / p+1 / p^{\prime}=1$. When $G$ is abelian, this inequality is just the usual Hausdorff-Young inequality [8, $\S 31.21]$, because $\left\|L_{f}\right\|_{p^{\prime}}$ is equal to the usual $L^{p^{\prime}}$-norm of the Fourier transform of $f$ in this case. To prove inequality (4), one must interpolate between the known cases where $p=1$ or $p=2$. In [9], Kunze used complex interpolation to this end. Recently, Peetre and Spaar [11] have used the real interpolation method to give a second proof of inequality (4).

Given a group $G$, and an index $p$ in the interval $(1,2)$, we let

$$
b_{p}(G)=\sup \left\{\left\|L_{f}\right\|_{p}: f \in L^{p}(G),\|f\|_{p} \leqq 1\right\} .
$$


Inequality (4) states that $b_{p}(G) \leqq 1$ in any case. In Theorem 2 of $\S 2$, we show that, for each index $p$ in the interval $(1,2)$, there exists a constant $B_{p}<1$ such that, if $G$ has no compact open subgroups, then $b_{p}(G) \leqq B_{p}$. In proving this, we reduce matters to the case where $p=4 / 3$, and then, following Young's original proof [18] of inequality (4) for the circle group with $p=4 / 3$, we show that

$$
b_{4 / 3}(G) \leqq c_{4 / 3,4 / 3}(G)^{1 / 2} ;
$$

the desired result then follows from Theorem 1.

A measurable function $f$ is called maximal if there is an index $p$ in the interval $(1,2)$ such that $0<\|f\|_{p}<\infty$, and such that equality holds in inequality (4). In Theorem 4 of $\S 3$, we show that a function is maximal if and only if it coincides, almost everywhere, with a multiple, by a nonzero constant, of a translate of a subcharacter. Again, we derive this statement from the corresponding assertion about maximal pairs of functions for convolution.

We now comment on the connections between these results and the work of other authors. First, Theorem 4 is not new, having been proved for the circle group by Hardy and Littlewood [5, Theorem 1], for all locally compact, abelian groups by Hewitt and Hirschmann [7], for all compact, nonabelian groups by Hewitt and Ross [8, Theorem 43.16], and in general by B. Russo [13], but the proof that we give here is new. Theorem 3 does not seem to have been stated before, although special cases of it follow easily from Theorem 4. We give two proofs of Theorem 3; the first proof is direct and elementary, while the second proof uses Theorem 4.

Theorems 1 and 2 do not seem to follow easily from Theorems 3 and 4. Consider the case of Theorem 2, for instance. If $G$ has no compact open subgroups, and $1<p<2$, then, by Theorem 4 , the only cases of equality in inequality (4) are the trivial ones where $\|f\|_{p}=0$ or $\infty$; it does not follow immediately, however, that $b_{p}(G)<1$ in this case, because it is not known whether the supremum in formula (5) must always be attained. By proving Theorem 2, we settle a conjecture due to Russo [13]. The basis for this conjecture was the theorem of K. I. Babenko [1] asserting that, if $p^{\prime}$ is even, then

$$
b_{p}(R)=\left[p^{1 / p} /\left(p^{\prime}\right)^{1 / p^{\prime}}\right]^{1 / 2} ;
$$

it follows easily that $b_{p}(R)<1$ whenever $1<p<2$. In [13], Russo showed that, if the group $G$ is central, or is a semidirect product of an abelian group $A$ and a compact group $K$ acting on $A$, and if $G$ has no compact open subgroups, then $b_{p}(G) \leqq b_{p}(R)$; other instances where this inequality holds are described in [12], and [14]. 
Recently, W. Beckner [2] has shown that Babenko's formula (6) holds for all indices $p$ in the interval $(1,2)$. In the same paper, Beckner used this result to compute $b_{p}(G)$ for all locally compact, abelian groups; in every case, $b_{p}(G)=b_{p}(R)^{n}$ for some integer $n$. It seems likely, therefore, that $b_{p}(G) \leqq b_{p}(R)$ whenever $G$ has no compact open subgroups. The methods of the present paper do not yield this inequality, but they do permit us to prove Theorem 2 for all unimodular groups.

The constants $c_{p, q}\left(R^{n}\right)$ have recently been computed by Beckner [2], and by Brascamp and Lieb [3]. Again, the methods of the present paper do not give us the exact values of the constants $c_{p, q}(G)$, but these methods are elementary, and they yield the conclusion of Theorem 1 for all unimodular groups.

2. Upper bounds for $c_{p, q}(G)$ and $b_{p}(G)$. In this section, we prove the two theorems stated below.

THEOREM 1. Let $(p, q)$ be an internal pair of indices. Then there exists a constant $C_{p, q}<1$ such that, if $G$ is a locally compact, unimodular group with no compact open subgroups, then $c_{p, q}(G) \leqq$ $C_{p, q}$.

THEOREM 2. Let $1<p<2$. Then there exists a constant $B_{p}<1$ such that, if $G$ is a locally compact unimodular group with no compact open subgroups, then $b_{p}(G) \leqq B_{p}$.

Proofs. Both theorems follow easily from the special case of Theorem 1 where $p=q=4 / 3$.

We first show how to derive Theorem 2 from this case of Theorem 1. Let $f \in L^{4 / 3}(G)$; denote by $f^{*}$ the function given by the rule that $f^{*}(x)=\overline{f\left(x^{-1}\right)}$ for all $x$ in $G$. It is shown in [9, Theorem 4] that $\left(L_{f}\right)^{*}$, the adjoint of the operator $L_{f}$, is just $L_{\left(f^{*}\right)}$. Thus

$$
\begin{aligned}
\left\|L_{f}\right\|_{4}^{4} & =m\left(\left|L_{f}\right|^{4}\right)=m\left(\left[\left(L_{f}\right)^{*} L_{f}\right]^{2}\right) \\
& =m\left(\left[L_{\left(f^{*}\right)} L_{f}\right]^{2}\right)=m\left(\left[L_{\left(f^{*}\right) * f}\right]^{2}\right) \\
& =\left\|\left(f^{*}\right) * f\right\|_{2}^{2}, \text { by relation }(3) \\
& \leqq\left[c_{4 / 3,4 / 3}(G)\left\|f^{*}\right\|_{4 / 3}\|f\|_{4 / 3}\right]^{2} \\
& =c_{4 / 3,4 / 3}(G)^{2}\left(\|f\|_{4 / 3}\right)^{4},
\end{aligned}
$$

since $G$ is unimodular. The calculation above is just Young's original argument [18] adapted to this setting. By this calculation,

$$
b_{4 / 3}(G) \leqq c_{4 / 3,4 / 3}(G)^{1 / 2} .
$$

If $G$ has no compact open subgroups, then 


$$
b_{4 / 3}(G) \leqq\left(C_{4 / 3,4 / 3}\right)^{1 / 2},
$$

by Theorem 1; thus the case of Theorem 2 where $p=4 / 3$ holds with $B_{4 / 3}=\left(C_{4 / 3,4 / 3}\right)^{1 / 2}$.

The other cases of Theorem 2 follow by convexity arguments. Suppose, for instance, that $1<p<4 / 3$; the argument when $4 / 3<$ $p<2$ similar. Let $\theta=4(1-1 / p)$; then

$$
\frac{1}{p}=\frac{1-\theta}{1}+\frac{\theta}{4 / 3} \text {. }
$$

By Riesz convexity [4, p. 143; 8, Appendix E],

$$
b_{p}(G) \leqq b_{1}(G)^{1-\theta} b_{4 / 3}(G)^{\theta} .
$$

Now $b_{1}(G) \leqq 1$ in any case. If $G$ has no compact open subgroups, then $b_{4 / 3}(G) \leqq B_{4 / 3}$, and

$$
b_{p}(G) \leqq\left(B_{4 / 3}\right)^{\theta}=B_{p}, \text { say. }
$$

We now turn to the proof of Theorem 1 when $p=q=4 / 3$. Let $\lambda$ be a number in the interval $(0,1)$ for which there exist functions $g$ and $f$ in $L^{4 / 3}(G)$ such that

$$
\|g\|_{4 / 3}=\|f\|_{4 / 3}=1 \text {, and }\|g * f\|_{2}>\lambda \text {. }
$$

We will show that if $\lambda$ is sufficiently close to 1 , then $G$ must have a compact open subgroup.

We begin with a very special case, and show later that matters can be reduced to this case. Suppose that $g$ is the characteristic function $1_{K}$ of a set $K$, and that $f=g^{*}$. Then $\mu(K)$, the Haar measure of $K$, is equal to 1 , because $\|g\|_{4 / 3}=1$. Now

$$
\begin{aligned}
g * f(x) & =\int_{G} 1_{K^{-1}}\left(y^{-1} x\right) 1_{K}(y) d y \\
& =\mu(x K \cap K) .
\end{aligned}
$$

Clearly, $g * f$ is continuous, and $0 \leqq g * f \leqq 1$.

We also consider two auxiliary functions, letting $h=(g * f)^{1 / 2}$, and $\phi=1-g * f$. Then $g * f \leqq h$, and

$$
\begin{aligned}
\|h\|_{2}^{2} & =\int_{G} g * f(x) d x \\
& =\|g\|_{1}\|f\|_{1}=1 .
\end{aligned}
$$

Given two sets $E$ and $F$, denote by $E \sim F$ the set of all elements of $E$ that do not belong to $F$. For each element $x$ of $G$, let $\ell(x) g$ be the left translate by $x$ of the function $g$; that is, $[\digamma(x) g](y)=$ $g\left(x^{-1} y\right)$, for all $y$ in $G$. Then 


$$
\phi(x)=\mu(x K \sim K)=\frac{1}{2}\|\ell(x) g-g\|_{1} .
$$

It follows from the triangle inequality for $L^{1}(G)$ that

$$
\phi(x)-\phi(y) \leqq \phi(x y) \leqq \phi(x)+\phi(y),
$$

for all $x$ and $y$ in $G$.

Given an integer $j$ between 1 and 5 , let

$$
H_{j}=\{x \in G: \phi(x)<j / 6\} .
$$

Outside the set $H_{1}$, the inequalities $\phi \geqq 1 / 6$, and $g * f \leqq 5 / 6$ hold; therefore,

$$
(g * f)^{2} \leqq \frac{5}{6}(g * f)=\frac{5}{6} h^{2} \text { outside } H_{1},
$$

and

$$
\int_{G \sim H_{1}}(g * f)^{2} \leqq \frac{5}{6} \int_{G \sim H_{1}} h^{2} .
$$

Then by relation (7) and the fact that $\|h\|_{2}=1$,

$$
\begin{aligned}
\lambda^{2} & \leqq \int_{G \sim H_{1}}(g * f)^{2}+\int_{H_{1}}(g * f)^{2} \\
& \leqq \frac{5}{6} \int_{G \sim H_{1}} h^{2}+\int_{H_{1}} h^{2} \\
& =\frac{5}{6} \int_{G} h^{2}+\frac{1}{6} \int_{H_{1}} h^{2} \\
& =\frac{5}{6}+\frac{1}{6} \int_{H_{1}} h^{2}=1-\frac{1}{6} \int_{G \sim H_{1}} h^{2} .
\end{aligned}
$$

This has two consequences concerning the measures of the sets $H_{j}$. First,

$$
\int_{H_{1}} h^{2} \geqq 6\left(\lambda^{2}-\frac{5}{6}\right)=6 \lambda^{2}-5 ;
$$

since $h^{2} \leqq 1$ in any case,

$$
\mu\left(H_{1}\right) \geqq 6 \lambda^{2}-5 .
$$

Second,

$$
\int_{H_{5} \sim H_{1}} h^{2} \leqq \int_{G \sim H_{1}} h^{2} \leqq 6\left(1-\lambda^{2}\right) ;
$$

since $h^{2}=1-\phi \geqq 1 / 6$ on the set $H_{5} \sim H_{1}$,

$$
\mu\left(H_{5} \sim H_{1}\right) \leqq 36\left(1-\lambda^{2}\right) .
$$


Now suppose that $\lambda^{2}>41 / 42$. We claim that the set $H_{4} \sim H_{2}$ must be empty. Indeed, if $x \in H_{4} \sim H_{2}$, then, by relation (8), the set $x H_{1}$ is included in $H_{5} \sim H_{1}$. Hence $\mu\left(H_{5} \sim H_{1}\right) \geqq \mu\left(H_{1}\right)$, so that, by relations (9) and (10), $6 \lambda^{2}-5 \leqq 36\left(1-\lambda^{2}\right)$, and $\lambda^{2} \leqq 41 / 42$, contrary to our assumptions. This proves the claim.

We claim further that $H_{2}$ is a compact open subgroup of $G$. First, $H_{2}$ contains the identity element $e$, because $\phi(e)=0$. Next, $\phi\left(x^{-1}\right)=\phi(x)$ for all $x$, so that $\left(H_{2}\right)^{-1}=H_{2}$. Now $H_{2} \cdot H_{2} \subset H_{4}$, by relation (8); since $H_{4} \sim H_{2}$ is empty, $H_{2} \cdot H_{2} \subset H_{2}$. Thus $H_{2}$ is a subgroup of $G$. Furthermore, $H_{2}$ is open, because $\phi$ is continuous. Finally, since $g * f>2 / 3$ on $H_{2}$, and $\|g * f\|_{2}<\infty$, the open subgroup $\mathrm{H}_{2}$ has finite Haar measure, and is therefore compact.

We now drop the assumption that $g$ and $f$ have the special forms prescribed above, and we merely suppose that the relation (7) holds; when it is convenient, however, we may, without loss of generality, replace the pair of functions $(g, f)$ by another pair for which these relations hold. For instance, since $|g| *|f| \geqq|g * f|$, we can assume that $g \geqq 0$ and $f \geqq 0$. In this situation, let $h=\left(g^{4 / 3} * f^{4 / 3}\right)^{1 / 2}$. Then

$$
\begin{aligned}
\|h\|_{2}^{2} & =\left\|g^{4 / 3} * f^{4 / 3}\right\|_{1} \\
& =\left\|g^{4 / 3}\right\|_{1}\left\|f^{4 / 3}\right\|_{1} \\
& =1,
\end{aligned}
$$

by Fubini's theorem, the fact that $g$ and $f$ are nonnegative, and the normalizations in relation (7). The proof of Young's inequality for convolution in this case consists in showing that $g * f \leqq h$. Indeed,

$$
\begin{aligned}
g * f(x) & =\int_{G} f\left(y^{-1} x\right) g(y) d y \\
& =\int_{G}\left[f\left(y^{-1} x\right) g(y)\right]^{2 / 3}\left[f\left(y^{-1} x\right) g(y)\right]^{1 / 3} d y \\
& \leqq\left\{\int_{G}\left[f\left(y^{-1} x\right) g(y)\right]^{4 / 3} d y\right\}^{1 / 2}\left\{\int_{G}\left[f\left(y^{-1} x\right) g(y)\right]^{2 / 3} d y\right\}^{1 / 2} \\
& =h(x)\left\{\int_{G}\left[f\left(y^{-1} x\right) g(y)\right]^{2 / 3} d y\right\}^{1 / 2} .
\end{aligned}
$$

We used the Schwarz inequality in the third line above; applying this inequality again, we have that

$$
\left\{\int_{G}\left[f\left(y^{-1} x\right) g(y)\right]^{2 / 3} d y\right\}^{1 / 2} \leqq\left[\int_{G} f\left(y^{-1} x\right)^{4 / 3} d y\right]^{1 / 4}\left[\int_{G} g(y)^{4 / 3} d y\right]^{1 / 4}=1,
$$

because $G$ is unimodular. Thus $0 \leqq g * f \leqq h$, and $\|g * f\|_{2} \leqq\|h\|_{2}=1$. On the other hand, $\|g * f\|_{2}>\lambda\|h\|_{2}$, by relation (7), so that there exist points $a$ in $G$ such that 


$$
0<\lambda h(a)<g * f(a)<\infty .
$$

By replacing $g$ by an appropriate left translate of itself, we can arrange for this relation to hold with $a=e$, the identity element of $G$. Moreover, by passing to an equivalent Haar measure on $G$, and renormalizing $g$ and $f$, we can arrange that $h(e)=1$. To do this, we replace $d x$ by $c d x, g$ by $c^{-3 / 4} g$, and $f$ by $c^{-3 / 4} f$; then $h$ is replaced by $c^{-1 / 2} h$, and $g * f$ by $c^{-1 / 2}(g * f)$. The norms $\|g\|_{4 / 3},\|f\|_{4 / 3}$, $\|g * f\|_{2}$, and $\|h\|_{2}$ are unaffected by this change, as is the ratio $g * f(e) / h(e)$. In summary, we can assume that

$$
\|g\|_{4 / 3}=\|f\|_{4 / 3}=\|h\|_{2}=h(e)=1,
$$

that $g \geqq 0$ and $f \geqq 0$, and that

$$
\|g * f\|_{2}>\lambda, \quad \text { and } \quad g * f(e)>\lambda .
$$

Let $\varepsilon=1-\lambda$. The idea now is to show that, if $\varepsilon$ is sufficiently close to 0 , then, in the metric of $L^{4 / 3}(G)$, the functions $g$ and $f^{*}$ are both close to a constant multiple of the characteristic function of a certain set. This will allow us to reduce matters to the very special case considered earlier in the proof.

Consider the proof that $g * f(e) \leqq h(e)=1$. Since $g * f(e)>1-\varepsilon$, the two applications above of the Schwarz inequality must be fairly sharp. The near sharpness of second application implies that $\left\|g^{2 / 3}-\left(f^{*}\right)^{2 / 3}\right\|_{2} \leqq 2 \varepsilon^{1 / 2}$. Letting $k=\left(g f^{*}\right)^{1 / 3}$, we have that $\left\|k-g^{2 / 3}\right\|_{2}$ and $\left\|k-\left(f^{*}\right)^{2 / 3}\right\|_{2}$ are both at most $2 \varepsilon^{1 / 2}$. Let $\alpha=\|k\|_{2}$. The near sharpness of the first application of the Schwarz inequality implies that $\left\|k^{2}-k / \alpha\right\|_{2} \leqq(2 \varepsilon)^{1 / 2}$. Let $K$ be the set where $k>1 / 2 \alpha$; then $\left\|k-(1 / \alpha) 1_{K}\right\|_{2} \leqq 2(2 \varepsilon)^{1 / 2}$. Letting $\gamma$ be the ratio of inner products $\left(k, 1_{K}\right) /\left(1_{K}, 1_{K}\right)$, we also have that $\left\|k-\gamma 1_{K}\right\|_{2} \leqq 2(2 \varepsilon)^{1 / 2}$, and that $\left\|\gamma 1_{K}\right\|_{2} \leqq 1$. Thus, $\left\|g^{2 / 3}-\gamma 1_{K}\right\|_{2}$ and $\left\|\left(f^{*}\right)^{2 / 3}-\gamma 1_{K}\right\|_{2}$ are both at most $2(1+\sqrt{2}) \varepsilon^{1 / 2}$. Using the inequality $|a-b| \leqq\left|a^{2 / 3}-b^{2 / 3}\right| \cdot\left|a^{1 / 3}+b^{1 / 3}\right|$, and Holder's inequality, we have that $\left\|g-\gamma^{3 / 2} 1_{K}\right\|_{4 / 3}$ and $\left\|f^{*}-\gamma^{3 / 2} 1_{K}\right\|_{4 / 3}$ are both at most $4(1+\sqrt{2}) \varepsilon^{1 / 2}$.

Letting $g^{\prime}=\gamma^{3 / 2} 1_{K}$ and $f^{\prime}=\left(g^{\prime}\right)^{*}$, we have that $\left\|g^{\prime} * f^{\prime}-g * f\right\|_{2} \leqq$ $8(1+\sqrt{2}) \varepsilon^{1 / 2}$, so that

$$
\left\|g^{\prime} * f^{\prime}\right\|_{2}>1-\varepsilon-8(1+\sqrt{2}) \varepsilon^{1 / 2}=1-\delta, \text { say. }
$$

By replacing $g$ by $g^{\prime} /\left\|g^{\prime}\right\|_{4 / 3}$ and $f$ by $f^{\prime} /\left\|f^{\prime}\right\|_{4 / 3}$ and by passing to an equivalent Haar measure, we can arrange that $g=1_{K}$, and $f=g^{*}$, and that relations (7) hold with $\lambda=1-\delta$. This is the very special case considered above; hence $G$ must have a compact open subgroup if $(1-\delta)^{2}>41 / 42$. This inequality holds whenever $\varepsilon$ sufficiently small, that is, whenever the original value of $\lambda$ is sufficiently close 
to 1. This completes the proof of the case of Theorem 1 where $p=q=4 / 3$.

The other cases of the theorem follow by simple Riesz convexity arguments, which we omit. Our methods do not lead to optimal values for the numbers $B_{p}$ and $c_{p, q}$, but we can specify values for these numbers. We now do this for $C_{4 / 3,4 / 3}$. The inequality $(1-\delta)^{2}>$ $41 / 42$ holds if $\delta \leqq 1 / 84$, and the latter inequality holds if $\varepsilon=3 \times$ $10^{-7}$. We can therefore let

$$
C_{4 / 3,4 / 3}=1-3 \times 10^{-7} .
$$

Our value for $B_{4 / 3}$ is then $\left(C_{4 / 3,4 / 3}\right)^{1 / 2}<.9999999$. This is much larger than $b_{4 / 3}(R)$, which is approximately .937 .

3. Maximality. In this section, we characterize the maximal pairs of functions for convolution, and the individual functions that are maximal for the Hausdorff-Young inequality. Before stating our results, however, we consider the basic examples, and the operations that preserve maximality.

First, we check that if $\chi$ is a subcharacter, then the pair $(\chi, \chi)$ is maximal for all internal pairs of indices. Now

$$
\chi^{*} \chi(y)=\int_{G} \chi\left(z^{-1} y\right) \chi(z) d z
$$

Let $H$ be the compact open subgroup that is the support of $\chi$. Then the product $\chi\left(z^{-1} y\right) \chi(z)$ vanishes unless both $z$ and $z^{-1} y$ belong to $H$; in that case, $y \in H$ also, and $\chi\left(z^{-1} y\right) \chi(z)=\chi(y)$, because the restriction of $\chi$ to $H$ is a character. Thus $\chi^{*} \chi=\mu(H) \chi$. It follows easily that the pair $(\chi, \chi)$ is maximal for all internal pairs of indices.

Recall that, for each element $a$ of $G$, and each function $f$ on $G$, the left translate of $f$ by $a$ is the function $\ell(a) f$ defined by letting $\ell(\alpha) f(y)=f\left(a^{-1} y\right)$ for all $y$ in $G$; similarly the right translate of $f$ by $a$ is the function (a) $f$ defined by letting $z(a) f(y)=f\left(y a^{-1}\right)$ for all $y$ in $G$. Let us say that two pairs $(g, f)$ and $\left(g^{\prime}, f^{\prime}\right)$, of functions on $G$, are equivalent if there exist elements $a$ and $b$ of $G$, and nonzero constants $c$ and $d$ such that $g^{\prime}$ and $f^{\prime}$ coincide, almost everywhere, with $c \ell(a) g$ and $d_{z}(b) f$ respectively. It is easy to check that maximality is preserved by this notion of equivalence. In Theorem 3, below, we show that a pair of functions $(g, f)$ is maximal if and only if there is a subcharacter $\chi$ such that $(g, f)$ is equivalent to $(\chi, \chi)$.

Let us say that two functions $f$ and $f^{\prime}$, on $G$, are equivalent if $f^{\prime}$ coincides, almost everywhere, with a multiple, by a nonzero constant, of a right translate of $f$. It is easy to check that equiva- 
lence preserves maximality. It is shown in [13] that a function is maximal if and only if it is equivalent to a subcharacter; in Theorem 4, below, we give a new proof that every maximal function is equivalent to a subcharacter. Now left translation also preserves maximality, and it may be surprising that this operation does not have to be included in the characterization of maximal functions. This omission is possible, however, because every left translate of a subcharacter is also a right translate of a possibly different subcharacter. Indeed, given a subcharacter $\chi$, with support group $H$, and an element $a$ of $G$, let $\chi^{\prime}$ be the function $\ell(a)_{2}\left(a^{-1}\right) \chi$; then $\chi^{\prime}$ is a subcharacter, with support group $a H \alpha^{-1}$, and $\ell(a) \chi=z(\alpha) \chi^{\prime}$.

The following two theorems imply that, if a pair of functions is maximal for one internal pair of indices, then this pair of functions is maximal for all internal pairs of indices, and if a single function is maximal for one index in the interval $(1,2)$, then this functions is maximal for all indices in the interval $(1,2)$.

THeORem 3. A pair of functions $(g, f)$ is maximal if and only if there is a subcharacter $\chi$ such that the pair $(g, f)$ is equivalent to the pair $(\chi, \chi)$.

THEOREM 4. A function is maximal if and only if it is equivalent to a subcharacter.

Proofs. We have already seen that if $\chi$ is a subcharacter, then every pair of functions that is equivalent to the pair $(\chi, \chi)$ is maximal for all internal pairs of indices. To prove the converse, we start with a pair of measurable functions $(g, f)$, and an internal pair of indices $(p, q)$ such that

$$
0<\|g\|_{p}<\infty, 0<\|f\|_{q}<\infty, \text { and }\|g * f\|_{r}=\|g\|_{p}\|f\|_{q} ;
$$

we then have to find a subcharacter $\chi$ such that the pairs $(g, f)$ and $(\chi, \chi)$ are equivalent. In the course of the proof, we may, without loss of generality, replace the pair $(g, f)$ by an equivalent pair whenever this is convenient.

First, we consider the case where $g \geqq 0$ and $f \geqq 0$. We can assume that $\|g\|_{p}=\|f\|_{q}=1$. Let $h=\left(g^{p} * f^{q}\right)^{1 / r}$; then $\|h\|_{r}=1$. The proof of Young's inequality for convolution, in this case, consists in showing that $g * f \leqq h$ everywhere. Indeed

$$
\begin{aligned}
g * f(x) & =\int_{G} f\left(y^{-1} x\right) g(y) d y \\
& =\int_{G}\left[f\left(y^{-1} x\right)^{q} g(y)^{p}\right]^{1 / r} f\left(y^{-1} x\right)^{1-q / r} g(y)^{1-p / r} d y .
\end{aligned}
$$


The definition of $r$ implies that $1 / r+1 / p^{\prime}+1 / q^{\prime}=1$; also, $(1-q / r) p^{\prime}=$ $q$, and $(1-p / r) q^{\prime}=p$. Apply Holder's inequality, with indices $r, p^{\prime}$, and $q^{\prime}$, to obtain the estimate

$$
\begin{aligned}
g * f(x) & \leqq h(x)\left[\int_{G} f\left(y^{-1} x\right)^{q} d y\right]^{1 / p^{\prime}}\left[\int_{G} g(y)^{p} d y\right]^{1 / q^{\prime}} \\
& =h(x), \text { because }\|f\|_{q}=\|g\|_{p}=1
\end{aligned}
$$

Since the pair $(g, f)$ is maximal,

$$
\|g * f\|_{r}=1=\|h\|_{r},
$$

and we must have that $g * f=h$ almost everywhere. Let

$$
H=\{x \in G: 0<h(x)<\infty \text {, and } g * f(x)=h(x)\} .
$$

For each $x$ in $H$, the application above of Holder's inequality is sharp; the condition for sharpness [6, p. 140] is that

$$
\frac{f\left(y^{-1} x\right)^{q} g(y)^{p}}{\int_{G} f\left(y^{-1} x\right)^{q} g(y)^{p} d y}=\frac{f\left(y^{-1} x\right)^{q}}{\int_{G} f\left(y^{-1} x\right)^{q} d y}=\frac{g(y)^{p}}{\int_{G} g(y)^{p} d y},
$$

for almost all $y$ in $G$. To be precise, this condition is necessary for sharpness when $1<r, p^{\prime}, q^{\prime}<\infty$, which is the case here, because the pair $(p, q)$ is internal. Since $\|f\|_{q}=\|g\|_{p}=1$, we can rewrite condition (12) as

$$
\frac{f\left(y^{-1} x\right)^{q} g(y)^{p}}{h(x)^{r}}=f\left(y^{-1} x\right)^{q}=g(y)^{p}, \text { for almost all } y \text {. }
$$

By translating $g$ appropriately, we can arrange that $e \in H$; moreover, by passing to an equivalent Haar measure on $G$, and renormalizing $g$ and $f$, we can arrange that $h(e)=1$. Then

$$
f\left(y^{-1}\right)^{q} g(y)^{p}=f\left(y^{-1}\right)^{q}=g(y)^{p}, \text { for almost all } y \text {. }
$$

Let $K=\{y \in G: g(y)>0\}$, and $K^{\prime}=\{y \in G: f(y)>0\}$. Then, for almost all $y$ in $K$,

$$
f\left(y^{-1}\right)^{q}=1, \quad \text { and } g(y)=f\left(y^{-1}\right)^{q / p}=1 ;
$$

in particular, $g=1_{K}$, almost everywhere. Similarly, for almost all $y$ in $K^{\prime}$,

$$
g\left(y^{-1}\right)=1, \text { and } f(y)=1 .
$$

Hence $f=g^{*}$ almost everywhere; we can assume that $g=1_{K}$, and that $f=1_{K}-1$ everywhere. Observe that $\mu(K)=1$, because $\|g\|_{p}=1$.

Now $g$ and $f$ both belong to $L^{1}(G) \cap L^{\infty}(G)$ so that $g * f$ and $h$ are both continuous; since $g * f=h$ almost everywhere, these func- 
tions coincide everywhere. Then $H$ is simply the set where $g * f>0$, so that $H$ is open. Since $f=g^{*}$, and $g=1_{K}$, formula (13) now reads that, for all $x$ in $H$,

$$
\frac{g\left(x^{-1} y\right) g(y)}{h(x)^{r}}=g\left(x^{-1} y\right)=g(y), \text { for almost all } y .
$$

Fix $x$ in $H$, and choose $y$ in $G$ so that these relations hold, and so that $g(y) \neq 0$; then $h(x)=g\left(x^{-1} y\right)^{1 / r}$. Since $h(x) \neq 0$ and $g$ takes the values 0 and 1 only, $h(x)=1$. That is, $g * f=h=1_{I I}$. Hence $\mu(H)=1$, because $\|h\|_{r}=1$. If $x \in H$, then, by formula (15), $\ell(x) g=g$ almost everywhere; conversely, if $\ell(x) g=g$ almost everywhere, then $g * f(x)>0$, and $x \in H$. Thus

$$
H=\{x: \measuredangle(x) g(y)=g(y) \text { for almost all } y \text { in } G\},
$$

and $H$ is a subgroup. Finally, since $\mu(H)<\infty$, the open subgroup $H$ is compact.

Consider relation (16). By Fubini's theorem, we have that, for almost all $y$ in $K$,

$$
g\left(x^{-1} y\right)=g(y), \text { for almost all } x \text { in } H .
$$

Fix an element $y$ of $K$ for which this relation holds. Then $g\left(x^{-1} y\right)=1$ for almost all $x$ in $H$. That is, almost all elements of the set $H y$ belong to $K$. Since $\mu(H y)=1=\mu(K)$, we conclude that $K$ is essentially the right coset $H y$ of $H$; we can assume that, in fact, $K=H y$. Let $H^{\prime}=y^{-1} H y$; then $K=y H^{\prime}$. Thus $g=\ell(y) 1_{H^{\prime}}$, and $f=\imath_{\imath}\left(y^{-1}\right) 1_{H^{\prime}}$, so that the pair $(g, f)$ is equivalent to the pair $\left(\mathbf{1}_{H^{\prime}}, 1_{H^{\prime}}\right)$.

Now we drop the assumption that $g$ and $f$ are nonnegative, but we continue to assume that they form a maximal pair for the internal pair of indices $(p, q)$. Since $|g * f| \leqq|g| *|f|$, the pair of functions $(|g|,|f|)$ is also maximal; by the analysis above, we can assume that $|g|=|f|=1_{I I}$, where $H$ is a compact open subgroup with $\mu(H)=1$. Since

$$
|g * f| \leqq|g| *|f|, \quad \text { and } \quad\|g * f\|_{r}=1=\||| g|*| f \mid\|_{r},
$$

we have that $|g * f|=|g| *|f|$ almost everywhere. But the functions $|g * f|$ and $|g| *|f|$ are continuous, and therefore coincide everywhere. In particular, $|g * f(e)|=|g| *|f|(e)=1$, and we may assume that $g * f(e)=1$.

Let $\phi=g * f$. To complete this proof of Theorem 3, we will show that $\phi$ is a subcharacter, and that the pair $(g, f)$ is equivalent to the pair $(\phi, \phi)$. As noted above, $\phi$ is continuous and supported by $H$. Fix $x$ in $H$; then, because $|g * f(x)|=|g| *|f|(x)$, we have equality in the inequality 


$$
\left|\int_{G} f\left(y^{-1} x\right) g(y) d y\right| \leqq \int_{G}\left|f\left(y^{-1} x\right) g(y)\right| d y .
$$

This can happen only if the integrand on the left has essentially constant argument on its support; this argument must then be the same as that of $g * f(x)$. Since $|g|=|f|=1_{H}$, we conclude, for each $x$ in $H$, that

$$
f\left(y^{-1} x\right) g(y)=\phi(x)
$$

for almost all $y$ in $H$. By Fubini's theorem, there exists a point $y$ in $H$ such that relation (17) holds for almost all $x$ in $H$. Fix such a point $y$. Then, as $x$ runs through $H$, the product $z=y^{-1} x$ also runs through $H$, and we conclude that

$$
f(z)=\phi(y z) / g(y),
$$

for almost all $z$ in $H$. We can modify $f$ so that this relation holds for all $z$ in $H$; then $f$ is continuous. Next, by relation (17), with $x=e$,

$$
g(y)=\phi(e) / f\left(y^{-1}\right)=\overline{f\left(y^{-1}\right)},
$$

for almost all $y$ in $H$. Again, we can modify $g$ so that this relation holds for all $y$ in $H$, that is, so that $g=f^{*}$. Then $f, g$, and $\phi$ are all continuous, and relation (17) holds for all $x$ and $y$ in $H$. We replace $y$ by $y^{-1}$ in this relation, and use the fact that $g\left(y^{-1}\right)=1 / f(y)$ to conclude that

$$
\phi(x)=\frac{f(y x)}{f(y)} \quad \text { for all } x \text { and } y \text { in } H .
$$

Let $x$ and $z$ belong to $H$. Then

$$
\phi(x z)=\frac{f(e x z)}{f(e)}=\frac{f(x z)}{f(z)} \cdot \frac{f(e z)}{f(e)}=\phi(x) \phi(z) .
$$

Thus $\phi$ is a subcharacter. By relation (18), with $y=e$, we have that $f=f(e) \phi$. Then

$$
g=f^{*}=\overline{f(e)} \phi^{*}=\overline{f(e)} \phi .
$$

This completes the proof of Theorem 3 .

We now consider the proof of Theorem 4. It is shown in [13, $p$, 295] that every subcharacter is maximal, for all indices in the interval $(1,2)$; it follows that every function that is equivalent to a subcharacter is maximal for all such indices. To prove the converse, let $1<p<2$, let $f \in L^{p}(G)$, and suppose that $\left\|L_{f}\right\|_{p^{\prime}}=\|f\|_{p} \neq 0$. Suppose first that $p=4 / 3$. Then Young's argument, as presented 
in the proof of Theorem 1, shows that the pair of functions $\left(f^{*}, f\right)$ is maximal for the pair of indices $(4 / 3,4 / 3)$; by Theorem 3 , the function $f$ is equivalent to subcharacter. Now suppose that $p \neq 4 / 3$, and let $f^{\prime}=|f|^{3 p / 4} \operatorname{sgn} f$. Evidently, $f^{\prime} \in L^{4 / 3}(G)$, and $\left\|f^{\prime}\right\|_{4 / 3} \neq 0$; the argument in [13, pp. 295-296] shows that $\left\|L_{f^{\prime}}\right\|_{4}=\left\|f^{\prime}\right\|_{4 / 3}$. Hence $f^{\prime}$ is equivalent to a subcharacter; then $f$ is equivalent to the same subcharacter. This completes the proof of Theorem 4 .

The problem of characterizing maximal pairs of functions has received less attention than the problem of characterizing functions that are maximal for the Hausdorff-Young theorem. It has been known for a long time, however, that there are no maximal pairs of functions on the real line [6, Inequality 280].

We now outline a second proof of the difficult half of Theorem 3. Suppose that $(g, f)$ is a maximal pair of functions, for the internal pair of indices $(p, q)$. Let $g^{\prime}=|g|^{3 p / 4} \operatorname{sgn} g$, and $f^{\prime}=|f|^{3 q / 4} \operatorname{sgn} f$; then the maximum modulus theorem can be used, as in [13, pp. 295-296] to show that the pair of functions $\left(g^{\prime}, f^{\prime}\right)$ is maximal for the pair of indices $(4 / 3,4 / 3)$. By Young's argument, as in the proof of Theorem 1, the functions $f^{\prime}$ and $\left(g^{\prime}\right)^{*}$ are both maximal in $L^{4 / 3}(G)$. Now Theorem 4 is already known [13]; so, we can use it to conclude that there exist subcharacters $\chi$ and $\phi$ such that $\left(g^{\prime}\right)^{*}$ is equivalent to $\chi$, and $f^{\prime}$ is equivalent to $\phi$. Then the pairs $\left(g^{\prime}, f^{\prime}\right)$ and $(\chi, \phi)$ are equivalent; hence so are the pairs $(g, f)$ and $(\chi, \phi)$. Let $H$ and $K$ be the support groups for $\chi$ and $\phi$ respectively; then the pair of functions $\left(1_{H}, 1_{K}\right)$ is maximal for the indices $(4 / 3,4 / 3)$. Consider the proof, in $\S 2$, of Young's inequality for convolution in this case. The second application of the Schwarz inequality there must be sharp for almost all $x$ in the support of $1_{I I} * 1_{K}$; hence, for such $x$, the function $\ell(x) 1_{K}$ must be a constant multiple of $1_{H}$. This means that the subgroups $H$ and $K$ coincide. This fact implies that if $\chi \neq \phi$, then $\chi * \phi=0$. Hence the only way for the pair $(\chi, \phi)$ to be maximal is for the two characters to coincide. Thus the pairs $(g, f)$ and $(\chi, \chi)$ are equivalent.

Theorems 3 and 4 suggest the following questions.

1. Is it true for all unimodular groups $G$ and all indices $p$ in the interval $(1,2)$ that there exist functions $f$ in $L^{p}(G)$ such that

$$
\left\|L_{f}\right\|_{p^{\prime}}=b_{p}(G)\|f\|_{p} \neq 0 ?
$$

2. Is it also true that, for each internal pair of indices $(p, q)$, there exist functions $g$ and $f$ such that

$$
0<\|g * f\|_{r}=c_{p, q}(G)\|g\|_{p}\|f\|_{q}<\infty ?
$$

3. For what functions do relations (19) and (20) hold? 
The results in this section provide answers to these questions when $b_{p}(G)=c_{p, q}(G)=1$, that is, when $G$ has a compact, open subgroup. It is known $[2,3]$ that the first two questions also have affirmative answers when $G=R^{n}$. It is also known, when $G=R^{n}$, that relation (20) holds if and only if the functions $g$ and $f$ are both obtained from a gaussian function $h$ and a character $\chi$ by translating the product $\chi \cdot h$, and multiplying it by a nonzero constant [3, Theorem 13]. This in turn leads to the same characterization of the functions for which relation (19) holds, in the special case where $G=R^{n}$, and $p^{\prime}$ is even.

\section{REFERENCES}

1. K. I. Babenko, On an inequality in the theory of Fourier integrals, Isz. Akad. Nauk. SSSR, Ser. Mat., 25 (1961), 531-542; English translation, Amer. Math. Soc. Transl. (2) 44, 115-128.

2. W. Beckner, Inequalities in Fourier analysis, Ann. of Math., 102 (1975), 159-182.

3. H. J. Brascamp and E. H. Lieb, Best constants in Young's inequality, its converse, and its generalization to more than three functions, Advances in Math., 20 (1976), 151-173.

4. C. F. Dunkl and D. E. Ramirez, Topics in Harmonic Analysis, Appleton-CenturyCrofts, New York, 1971.

5. G. H. Hardy and J. E. Littlewood, Some new properties of Fourier constants, Math. Ann., 97 (1927), 159-209.

6. G. H. Hardy, J. E. Littlewood and G. Polya, Inequalities, first edition, Cambridge University Press, 1934.

7. E. Hewitt and I. I. Hirschman Jr., A maximum problem in harmonic analysis, Amer. J. Math., 76 (1954), 839-852.

8. E. Hewitt and K. A. Ross, Abstract Harmonic Analysis, Springer-Verlag, Heidelberg, 1963,1970 , two volumes.

9. R. A. Kunze, $L_{p}$ Fourier transforms on locally compact unimodular groups, Trans. Amer. Math. Soc., 89 (1958), 519-540.

10. E. Nelson, Notes on non-commutative integration, J. Functional Anal., 15 (1974), 103-116.

11. J. Peetre and G. Sparr, Interpolation and non-commutative integration, Ann. Math. Pura Appl., (4) 104 (1975), 187-207.

12. B. Russo, On the Hausdorff-Young theorem for integral operators, Pacific J. Math., 68 (1977), 241-253.

13. - The norm of the $L^{p}$-Fourier transform on unimodular groups, Trans. Amer. Math. Soc., 192 (1974), 293-305.

14. - The norm of the $L^{p}$-Fourier transform II, Canad. J. Math., 28 (1976), $1121-1131$.

15. I. E. Segal, A non-commutative extension of abstract integration, Ann. of Math., 57 (1953), 401-457; correction, 58 (1953), 595-596.

16. W. F. Stinespring, Integration theorems for gages and duality for unimodular groups, Trans. Amer. Math. Soc., 90 (1959), 15-56.

17. A. Weil, $L^{\prime}$ Intégration dans les groupes topologiques et ses applications, Actualités Sci et. Ind. 869, 1145, Hermann et Cie, 1941 and 1951.

18. W. H. Young, On the multiplication of successions of Fourier constants, Proc. Roy. Soc. London, Ser. A, 87 (1912), 331-339.

Received January 27, 1977. Research partially support by National Research Council of Canada grant number 4822 . 



\title{
PACIFIC JOURNAL OF MATHEMATICS
}

\section{EDITORS}

\author{
RICHARD ARENS (Managing Editor) \\ University of California \\ Los Angeles, CA 90024 \\ Charles W. Curtis \\ University of Oregon \\ Eugene, OR 97403 \\ C. C. MOORE \\ University of California \\ Berkeley, CA 94720
}

\section{J. DUGUNDJI}

Department of Mathematics

University of Southern California

Los Angeles, CA 90007

R. FINN and J. MILGRAM

Stanford University

Stanford, CA 94305

\section{ASSOCIATE EDITORS}
E. F. BECKENBACH
B. H. NeumanN
F. WOLF
K. YoSHIDA

\section{SUPPORTING INSTITUTIONS}

UNIVERSITY OF BRITISH COLUMBIA

CALIFORNIA INSTITUTE OF TECHNOLOGY

UNIVERSITY OF CALIFORNIA

MONTANA STATE UNIVERSITY

UNIVERSITY OF NEVADA, RENO

NEW MEXICO STATE UNIVERSITY

OREGON STATE UNIVERSITY

UNIVERSITY OF OREGON

OSAKA UNIVERSITY

\author{
UNIVERSITY OF SOUTHERN CALIFORNIA \\ STANFORD UNIVERSITY \\ UNIVERSITY OF HAWAII \\ UNIVERSITY OF TOKYO \\ UNIVERSITY OF UTAH \\ WASHINGTON STATE UNIVERSITY \\ UNIVERSITY OF WASHINGTON \\ $\stackrel{*}{*} \stackrel{*}{*} \stackrel{*}{*}$ AMERICAN MATHEMATICAL SOCIETY
}

The Supporting Institutions listed above contribute to the cost of publication of this Journal, but they are not owners or publishers and have no responsibility for its content or policies.

Mathematical papers intended for publication in the Pacific Jaurnal of Mathematics should be in typed form or offset-reproduced, (not dittoed), double spaced with large margins. Please do not use built up fractions in the text of your manuscript. You may however, use them in the displayed equations. Underline Greek letters in red, German in green, and script in blue. The first paragraph or two must be capable of being used separately as a synopsis of the entire paper. Items of the bibliography should not be cited there unless absolutely necessary, in which case they must be identified by author and Journal, rather than by item number. Manuscripts, in triplicate, may be sent to any one of the editors. Please classify according to the scheme of Math. Reviews, Index to Vol. 39. All other communications should be addressed to the managing editor, or Elaine Barth, University of California, Los Angeles, California, 90024.

The Pacific Journal of Mathematics expects the author's institution to pay page charges, and reserves the right to delay publication for nonpayment of charges in case of financial emergency.

100 reprints are provided free for each article, only if page charges have been substantially paid. Additional copies may be obtained at cost in multiples of 50 .

The Pacific Journal of Mathematics is issued monthly as of January 1966. Regular subscription rate: $\$ 7200$ a year (6 Vols., 12 issues). Special rate: $\$ 36.00$ a year to individual members of supporting institutions.

Subscriptions, orders for back numbers, and changes of address should be sent to Pacific Journal of Mathematics, 103 Highland Boulevard, Berkeley, California, 94708.

PUBLISHED BY PACIFIC JOURNAL OF MATHEMATICS, A NON-PROFIT CORPORATION

Printed at Kokusai Bunken Insatsusha (International Academic Printing Co., Ltd.). 8-8, 3-chome, Takadanobaba, Shinjuku-ku, Tokyo 160, Japan.

Copyright (C) 1975 by Pacific Journal of Mathematics Manufactured and first issued in Japan 


\section{Pacific Journal of Mathematics}

Vol. 72, No. 2

February, 1977

George E. Andrews, Plane partitions. II. The equivalence of the

Bender-Knuth and MacMahon conjectures ................. 283

Lee Wilson Badger, An Ehrenfeucht game for the multivariable quantifiers

of Malitz and some applications ......................... 293

Wayne C. Bell, A decomposition of additive set functions ............ 305

Bruce Blackadar, Infinite tensor products of $C^{*}$-algebras ............. 313

Arne Brøndsted, The inner aperture of a convex set .............. 335

N. Burgoyne, Finite groups with Chevalley-type components........... 341

Richard Dowell Byrd, Justin Thomas Lloyd and Roberto A. Mena, On the retractability of some one-relator groups .....................

Paul Robert Chernoff, Schrödinger and Dirac operators with singular potentials and hyperbolic equations .................... 361

John J. F. Fournier, Sharpness in Young's inequality for convolution ....... 383

Stanley Phillip Franklin and Barbara V. Smith Thomas, On the metrizability

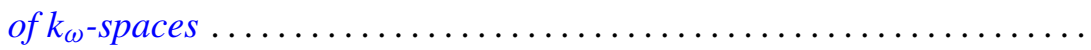

David Andrew Gay, Andrew McDaniel and William Yslas Vélez, Partially normal radical extensions of the rationals .................... 403

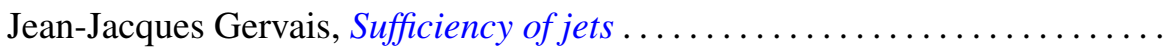

Kenneth R. Goodearl, Completions of regular rings. II . .

Sarah J. Gottlieb, Algebraic automorphisms of algebraic groups with stable maximal tori

Donald Gordon James, Invariant submodules of unimodular Hermitian forms.....

J. Kyle, $W_{\delta}(T)$ is convex.

Ernest A. Michael and Mary Ellen Rudin, A note on Eberlein compacts ...

Ernest A. Michael and Mary Ellen Rudin, Another note on Eberlein compacts ....

Thomas Bourque Muenzenberger and Raymond Earl Smithson, Fixed point theorems for acyclic and dendritic spaces.

Budh Singh Nashier and A. R. Rajwade, Determination of a unique solution of the quadratic partition for primes $p \equiv 1(\bmod 7)$.

Frederick J. Scott, New partial asymptotic stability results for nonlinear ordinary differential equations ....................

Frank Servedio, Affine open orbits, reductive isotropy groups, and dominant gradient morphisms; a theorem of Mikio Sato..........

D. Suryanarayana, On the distribution of some generalized square-full integers.................................. 